UMD-PP-02-019

JHU-TIPAC-2001-05

\title{
Anomaly Mediated Supersymmetry Breaking in Four Dimensions, Naturally
}

\author{
Markus A. Luty \\ Department of Physics, University of Maryland \\ College Park, Maryland 20742, USA \\ mluty@physics.umd.edu \\ Raman Sundrum \\ Department of Physics and Astronomy, Johns Hopkins University \\ Baltimore, Maryland 21218, USA \\ sundrum@pha.jhu.edu
}

\begin{abstract}
We present a simple four-dimensional model in which anomaly mediated supersymmetry breaking naturally dominates. The central ingredient is that the hidden sector is near a strongly-coupled infrared fixed-point for several decades of energy below the Planck scale. Strong renormalization effects then sequester the hidden sector from the visible sector. Supersymmetry is broken dynamically and requires no small input parameters. The model provides a natural and economical explanation of the hierarchy between the supersymmetry-breaking scale and the Planck scale, while allowing anomaly mediation to address the phenomenological challenges posed by weak scale supersymmetry. In particular, flavor-changing neutral currents are naturally near their experimental limits.
\end{abstract}




\section{Introduction}

Anomaly mediated supersymmetry breaking (AMSB) [1, 2] is a general supergravity mechanism that is tightly constrained by local supersymmetry. AMSB may play an important role in solving the major phenomenological problems of weak scale supersymmetry (SUSY): the flavor, $\mu$, and gaugino mass problems. For example, Ref. [3] describes a complete and very plausible extended supersymmetric standard model where anomaly mediation is the main ingredient in solving these problems and leads to a realistic and distinctive spectrum. Refs. [4 describe other proposals for weak scale AMSB.

In order for AMSB to dominate in the observable sector, SUSY breaking must originate in a special type of hidden sector. A general hidden sector model has the form

$$
\mathcal{L}=\mathcal{L}_{\text {SUGRA }}+\mathcal{L}_{\text {visible }}+\mathcal{L}_{\text {hidden }}+\mathcal{L}_{\text {mixed }}
$$

where the first three terms are self-explanatory, while $\mathcal{L}_{\text {mixed }}$ contains Planck-suppressed terms involving both visible and hidden fields that cannot naturally be forbidden by symmetries. AMSB in the visible sector arises from minimal coupling to supergravity, in particular the auxiliary scalar field in the minimal formulation. By supercovariance, this scalar couples via visible mass scales, in particular the renormalization scale associated with the scale anomaly in radiative corrections (hence 'anomaly mediation'). Therefore, supersymmetry breaking effects arising from AMSB are suppressed by loop factors. In general hidden sector models, larger visible SUSY breaking can arise directly from the hidden sector through terms in $\mathcal{L}_{\text {mixed }}$. Therefore, in order for AMSB to dominate $\mathcal{L}_{\text {mixed }}$ must be strongly suppressed.t That is, the hidden and visible sectors are 'sequestered'.

In Refs. [1, [] it was shown that sequestering can be achieved if the visible and hidden sectors are localized on different 3-branes separated in extra dimensions. Recently, we demonstrated that highly warped supersymmetric anti de Sitter space (AdS) compactifications could be stabilized with sufficient sequestering [6]. AdS/conformal field theory (CFT) duality [7] applied to such compactifications [8] then suggests that sequestering can also arise in a purely $4 \mathrm{D}$ context with the help of strongly coupled conformal dynamics. In Ref. [9], we showed that sequestering in fact occurs in a large class of supersymmetric CFT's. We also presented a specific model incorporating SUSY breaking of the required type. This model is technically natural, but

\footnotetext{
${ }^{1}$ Note that this is true in superspace, but not after component level field redefinitions to go to Einstein frame. See Ref. [3].
} 
it requires several unexplained small numbers. In this paper, we will present a very simple and plausible model of conformal sequestering, in which all large hierarchies are dynamically generated. Using 'naïve dimensional analysis' to estimate the strong interaction coefficients, we find that the model easily gives enough sequestering so that anomaly mediation dominates, and flavor-changing neutral currents are near their experimental limits.

The basic structure of our model is as follows. The central component of $\mathcal{L}_{\text {hid }}$ is a SUSY theory that is near a strongly-coupled conformal fixed point below the Planck scale. The infrared approach to the fixed point is governed by an order one critical exponent $\beta_{*}^{\prime}$. Imposing certain exact hidden symmetries restricts the hidden sector factors in $\mathcal{L}_{\text {mixed }}$ to have the same form as the operators in $\mathcal{L}_{\text {hid }}$. Because of this, the operators in $\mathcal{L}_{\text {mixed }}$ can be viewed as perturbations of hidden sector couplings with visible sector coefficients. All such perturbations are suppressed by $(\mu / M)^{\beta_{*}^{\prime}}$ as the hidden sector approaches the fixed point, where $\mu$ is the renormalization scale and $M$ is the Planck scale. This is the conformal sequestering mechanism.

Superconformal field theories naturally have a moduli space. They are exactly superconformal only at the origin of moduli space, but away from the origin, superconformal invariance is spontaneously broken. The degeneracy of these vacua is lifted by weak, even technically irrelevant perturbations to the fixed point theory. We use such effects to generate an effective potential for the hidden moduli space which stabilizes the moduli away from the origin with a SUSY breaking vacuum energy. The small numbers needed to ensure that the SUSY breaking scale and the moduli VEVs are hierarchically smaller than the Planck scale are naturally generated by non-perturbative effects.

For an earlier application of strong conformal dynamics (in the visible sector) to supersymmetric model-building see Ref. [10].

\section{The Model}

In this paper, we focus on the hidden sector and the mechanism for sequestering from the visible sector. The visible sector can be any theory for which AMSB yields an acceptable phenomenology. For now, we will restrict ourselves to global SUSY. In Section 4, we will consider the SUGRA corrections to the effective potential, which are important for modulus stabilization and cancelling the cosmological constant.

Our model of the hidden sector consists of two supersymmetric QCD (SQCD) subsectors: a $S U(2)$ gauge theory with 4 flavors (8 fundamentals) $T^{J a}(J=1, \ldots, 4$; $a=1,2)$, denoted by $\mathrm{SQCD}_{2}$; and a $S U(3)$ gauge theory with 2 flavors $P^{a}, \bar{P}_{a}$ 
$(a=1,2)$, denoted by $\mathrm{SQCD}_{3}$. Throughout the paper we will suppress all gauge indices, and we will suppress the $a=1,2$ index when the meaning is clear. We impose the following symmetries on the hidden sector: permutations of the $T^{J}$, multiplication of any of the $T^{J}$ by -1 , charge conjugation for $\mathrm{SQCD}_{3}$, and a global $S U(2)$ symmetry acting on the $a=1,2$ index.'2 The theory has a superpotential invariant under these symmetries:

$$
W=\frac{\lambda}{M} \sum_{J}\left(T^{J} T^{J}\right)(\bar{P} P)+\frac{\lambda^{\prime}}{M} \sum_{J \neq K}\left(T^{J} T^{J}\right)\left(T^{K} T^{K}\right),
$$

where $M$ is the Planck scale. We will show that this simple model sequesters itself from the visible sector and has a local minimum that dynamically breaks SUSY.

The $\mathrm{SQCD}_{2}$ sector is at the self-dual point of Seiberg's conformal window 11 and we will assume that it starts near its IR fixed point coupling at the Planck scale. It is therefore strongly coupled. We assume the $\mathrm{SQCD}_{3}$ sector is weakly coupled at the Planck scale. We also assume that the superpotential couplings $\lambda$ and $\lambda^{\prime}$ are sufficiently small that they can be treated as perturbations of the $\mathrm{SQCD}_{2}$ fixed point.

The leading dangerous terms in $\mathcal{L}_{\text {mixed }}$ compatible with the hidden sector symmetries are

$$
\mathcal{L}_{\text {mixed }}(M)=\int d^{4} \theta\left[\frac{c^{j} k}{M^{2}} Q_{j}^{\dagger} Q^{k} \sum_{J} T_{J}^{\dagger} T^{J}+\frac{\left(c_{P}\right)^{j} k}{M^{2}} Q_{j}^{\dagger} Q^{k}\left(P^{\dagger} P+\bar{P}^{\dagger} \bar{P}\right)\right],
$$

where the $Q_{j}$ are visible chiral superfields. The danger is that for $c, c_{P}$ of order unity and containing SM flavor violation, flavor violating visible scalar masses will be generated upon SUSY breaking in the hidden sector that will dominate over the flavor-blind AMSB contributions. We will show that the $\mathrm{SQCD}_{2}$ conformal dynamics naturally suppresses the effects of $c, c_{P}$ at low energies, allowing AMSB to dominate the visible sector. Supergravity loops can contribute to mixed couplings, but they are dominant in the ultraviolet, so their leading effects can be absorbed into the $c$ coefficients.

\footnotetext{
${ }^{2}$ For readers concerned by quantum gravity violation of global symmetries: the $S U(2)$ group can be weakly gauged, or can be replaced by a suitable discrete subgroup.
} 


\section{Sequestering}

We first consider the limit $\lambda=\lambda^{\prime}=0$. In this limit the $\mathrm{SQCD}_{3}$ sector completely decouples and we can omit it from the discussion. The leading terms in $\mathcal{L}_{\text {mixed }}$ (see Eq. (2.2)) can be viewed as perturbations to the wavefunction of the hidden fields renormalized at the Planck scalep:

$$
\left(\mathcal{L}_{\text {hidden }}+\mathcal{L}_{\text {mixed }}\right)(M)=\int d^{4} \theta Z_{0} T^{\dagger} T+\left(\int d^{2} \theta \tau_{\text {hol }, 0} \operatorname{tr} W^{\alpha} W_{\alpha}+\text { h.c. }\right),
$$

where

$$
Z_{0}=z_{0}+\frac{c^{j} k}{M^{2}} Q_{j}^{\dagger} Q^{k}
$$

We will explain the role of $z_{0}$ below. $\tau_{\text {hol, } 0}$ is the holomorphic $S U(2)$ gauge coupling. The theory defined by Eq. (3.1) has only one physical coupling, namely the physical gauge coupling $\tau=1 / g^{2}$, given by

$$
\tau=\operatorname{Re}\left(\tau_{\mathrm{hol}}\right)-\frac{F}{8 \pi^{2}} \ln Z+\frac{N}{8 \pi^{2}} \ln \tau+f(\tau) .
$$

where $N$ is the number of colors and $F$ is the number of flavors; in our theory, $N=2$, $F=4$. Here $f(\tau)=$ constant $+\mathcal{O}\left(\tau^{-1}\right)$ parameterizes the scheme dependence. The perturbation Eq. (3.2) (holding $\tau_{\text {hol, } 0}$ fixed) therefore gives rise to a perturbation of the physical gauge coupling. Taking the derivative $d / d t$ of Eq. (3.3), where $t \equiv \ln \mu / M$, we obtain

$$
\beta(\tau)=\frac{\frac{b}{8 \pi^{2}}-\frac{F}{8 \pi^{2}} \gamma(\tau)}{1-\frac{N}{8 \pi^{2}} \frac{1}{\tau}-f^{\prime}(\tau)}, \quad b=3 N-F,
$$

where $\beta \equiv d \tau / d t, \gamma \equiv d \ln Z / d t$, and we have used $d \tau_{\text {hol }} / d t=b / 8 \pi^{2}$. In the 'NSVZ scheme' $f \equiv 0$, Eqs. (3.3) and (3.4) are the famous formulae of Refs. [12].

Because Eq. (3.2) is a perturbation to the UV gauge coupling, it is clear that it is irrelevant near the IR fixed point. This means that the effects of the perturbation $c^{j}{ }_{k} Q_{j}^{\dagger} Q^{k} / M^{2}$ are suppressed in the IR. This is the underlying mechanism for sequestering in this class of models [9].

${ }^{3}$ Note that without imposing the hidden-flavor symmetries discussed in Section 2, the mixed terms could be more general than this form. In this case we would encounter the difficulties discussed in Ref. [9].

${ }^{4}$ Note that $Z_{0}$ in Eq. (3.2) is a vector superfield. By 'analytic continuation into superspace' Eq. (3.3) can be interpreted as an equality of vector superfields 13. 
We now make this quantitative. Exactly at the fixed point, $\tau=\tau_{*}=$ constant, so $\gamma(\tau)=\gamma\left(\tau_{*}\right) \equiv \gamma_{*}=$ constant. Therefore (taking $Z_{*}(t=0)=1$ )

$$
Z_{*}(t)=e^{\gamma_{*} t} .
$$

The theory is at a fixed point despite the running of $Z$ because the running of $\tau_{\text {hol }}$ compensates so that $\beta\left(\tau_{*}\right)=0$. From Eq. (3.4) we see that this requires [11]

$$
\gamma_{*}=\frac{b}{F} .
$$

We now consider the perturbations about the fixed point. We expand the RG functions in $\Delta \tau \equiv \tau-\tau_{*}$ to first order to define critical exponents

$$
\begin{aligned}
& \beta(\tau) \simeq \beta_{*}^{\prime} \cdot \Delta \tau, \\
& \gamma(\tau) \simeq \gamma_{*}+\gamma_{*}^{\prime} \cdot \Delta \tau .
\end{aligned}
$$

We factor out the fixed point running by defining

$$
\Delta \ln Z \equiv \ln Z-\gamma_{*} t .
$$

Then we have

$$
\frac{d(\Delta \ln Z)}{d t}=\gamma_{*}^{\prime} \cdot \Delta \tau, \quad \frac{d(\Delta \tau)}{d t}=\beta_{*}^{\prime} \cdot \Delta \tau .
$$

Because of the relation Eq. (3.3), these equations are not independent. Using Eq. (3.3) we can write an $\mathrm{RG}$ equation for $\Delta \ln Z$ alone:

$$
\frac{d(\Delta \ln Z)}{d t}=\beta_{*}^{\prime}\left[\Delta \ln Z-\frac{8 \pi^{2}}{F} \Delta \tau_{\mathrm{hol}, 0}\right] .
$$

Here $\Delta \tau_{\text {hol }} \equiv \tau_{\text {hol }}-\tau_{\text {hol, },}$, and $\tau_{\text {hol, } *}$ satisfies Eq. (3.3) for $\tau=\tau_{*}, Z=Z_{*}$. The deviation from the fixed point in the UV is parameterized by $Z_{0} \neq 1$ (see Eq. (3.2) and Eq. (3.5)) and $\Delta \tau_{\text {hol }, 0} \neq 0$. From Eq. (3.3) we can see that these are not independent perturbations, so we can choose $\Delta \tau_{\mathrm{hol}, 0}=0$ and parameterize the perturbation by $Z_{0}$ alone. The solution to Eq. (3.11) is then simply

$$
\Delta \ln Z=e^{\beta_{*}^{\prime} t}(\Delta \ln Z)_{0} .
$$

$\beta_{*}^{\prime}$ is a strong-interaction critical exponent of order one. Since the dangerous terms in $\mathcal{L}_{\text {mixed }}$ are contained in $\Delta \ln Z_{0}$, this clearly shows the sequestering. 
Now we include the effects of $\lambda, \lambda^{\prime}$, and $\tau_{3}$. From now on we specialize to the case $F=2 N$ for the $\mathrm{SQCD}_{2}$ sector, so that $\gamma_{*}=\frac{1}{2}$. We must now include the additional mixed terms

$$
Z_{P, 0}=1+\frac{\left(c_{P}\right)^{j} k}{M^{2}} Q_{j}^{\dagger} Q^{k} .
$$

Because the $S U(3)$ sector is not a CFT, we expect at most an order 1 renormalization of $Z_{P}$. Since we are only interested in the order of magnitude of $Z_{P}$, we will simply use the approximation $Z_{P} \simeq Z_{P, 0}$. The mixed terms in Eq. (3.13) do not directly give rise to large visible soft masses because in our model the dominant source of SUSY breaking is in the $\mathrm{SQCD}_{2}$ sector.

However, we must determine the leading effects of the perturbation Eq. (3.13) on the $\mathrm{SQCD}_{2}$ sector. These can be studied in the RG equation for $\Delta \ln Z$ :

$$
\frac{d(\Delta \ln Z)}{d t}=\gamma_{*}^{\prime} \cdot \Delta \tau+\Delta \gamma\left(\tau, \tau_{3}, \lambda_{\text {phys }}, \lambda_{\text {phys }}^{\prime}\right),
$$

where

$$
\begin{aligned}
& \lambda_{\text {phys }}=\frac{\lambda \mu}{M Z Z_{P}}=\frac{\lambda e^{t / 2} e^{-\Delta \ln Z}}{Z_{P}}, \\
& \lambda_{\text {phys }}^{\prime}=\frac{\lambda^{\prime} \mu}{M Z^{2}}=\lambda^{\prime} e^{-2 \Delta \ln Z} .
\end{aligned}
$$

While $\Delta \gamma$ is a small perturbation in Eq. (3.14), it becomes comparable to the first term on the right-hand side in the IR. We must show that this does not spoil sequestering. Since we are expanding around the fixed point we can set $\tau=\tau_{*}$ in $\Delta \gamma$. We will use Eq. (3.14) only in the regime where the $\mathrm{SQCD}_{3}$ sector is unbroken and weakly coupled. In this regime, we can neglect the running due to $\tau_{3}$. The leading terms are therefore

$$
\frac{d(\Delta \ln Z)}{d t}=\gamma_{*}^{\prime} \cdot \Delta \tau+\frac{\left|\lambda_{\text {phys }}\right|^{2}}{\rho^{4}}+\frac{\left|\lambda_{\text {phys }}^{\prime}\right|^{2}}{\rho^{4}} .
$$

Because of the $\mathrm{SQCD}_{2}$ strong interaction uncertainties, we cannot compute the coefficients of the last two terms precisely, but we have estimated their order of magnitude using 'naive dimensional analysis' (NDA) [14, 15]. Here, and later in the paper, we will give our NDA estimates in terms of

$$
\rho \sim 4 \pi .
$$

Separate order one uncertainties should then be ascribed to different terms, but these will not be written explicitly. 
Once again, we would like to use Eq. (3.3) to eliminate $\Delta \tau$ on the right-hand side of Eq. (3.16) in favor of $\Delta \ln Z$. In the presence of the additional couplings $\lambda, \lambda^{\prime}$, and $\tau_{3}$ Eq. (3.3) remains true, but the scheme dependent function $f$ is in general a function of all the couplings. However, we can always choose a scheme where $f$ is a function of $\tau$ alone. In such a scheme we have

$$
\frac{d(\Delta \ln Z)}{d t}=\beta_{*}^{\prime} \Delta \ln Z+\frac{\left|\lambda_{\text {phys }}\right|^{2}}{\rho^{4}}+\frac{\left|\lambda_{\text {phys }}^{\prime}\right|^{2}}{\rho^{4}} .
$$

The last two terms on the right-hand side are subdominant perturbations compared to the first term unless $\Delta \ln Z$ is small. Therefore we can approximate the last two terms using Eq. (3.15) in the limit $\Delta \ln Z \rightarrow 0$. Also $Z_{P}$ runs only perturbatively, so we can approximate $Z_{P} \simeq Z_{P, 0}$. We then obtain the approximate solution

$$
\begin{gathered}
\Delta \ln Z \simeq e^{\beta_{*}^{\prime} t}(\Delta \ln Z)_{0}+\frac{|\lambda|^{2}}{\rho^{4} Z_{P, 0}^{2}} \frac{e^{\beta_{*}^{\prime} t}-e^{t}}{\beta_{*}^{\prime}-1} \\
+\frac{\left|\lambda^{\prime}\right|^{2}}{\rho^{4}} \frac{e^{\beta_{*}^{\prime} t}-1}{\beta_{*}^{\prime}} .
\end{gathered}
$$

The first two terms contain mixed terms, but are sequestered, while the third term is not sequestered, but contains no mixed terms. Therefore, all mixed terms are suppressed in this model provided that there is a sufficiently large range of scales for which the $\mathrm{SQCD}_{2}$ sector is near the fixed point. In fact, the above perturbations due to $\lambda, \lambda^{\prime}$ have subdominant effects to others we will later identify and to $\Delta \ln Z$.

It is convenient to summarize the RG near the fixed point by writing the effective lagrangian

$$
\begin{aligned}
\mathcal{L} \simeq & \int d^{4} \theta\left[\mu^{1 / 2}(1+\Delta \ln Z) \tilde{T}^{\dagger} \tilde{T}+Z_{P, 0}\left(P^{\dagger} P+\bar{P}^{\dagger} \bar{P}\right)\right] \\
& +\int d^{2} \theta\left[\frac{\lambda}{M^{1 / 2}} \sum_{J}\left(\tilde{T}^{J} \tilde{T}^{J}\right)(\bar{P} P)+\lambda^{\prime} \sum_{J \neq K}\left(\tilde{T}^{J} \tilde{T}^{J}\right)\left(\tilde{T}^{K} \tilde{T}^{K}\right)\right]+\text { h.c. }
\end{aligned}
$$

+ gauge kinetic terms,

where we have defined the rescaled fields [9],

$$
\tilde{T} \equiv \frac{T}{M^{1 / 4}}
$$

This rescaling removes the leading $M$ dependence of the lagrangian, and makes the canonical dimension of the $\tilde{T}$ fields the same as their fixed-point scaling dimension in chiral operators. 


\section{Supersymmetry Breaking}

We now determine the vacuum in this theory. We will show that there is a locally stable vacuum with broken SUSY at $T \neq 0$.

In the absence of the superpotential couplings Eq. (2.1), the $\mathrm{SQCD}_{2}$ theory has 13 independent moduli, which can be parameterized by the $S U(2)$ gauge invariant 'meson' operators of the form $T^{J a} T^{K b}$ subject to classical constraints. Away from the origin of moduli space the superpotential couplings proportional to $\lambda^{\prime}$ reduce the moduli space to a single flat direction, which we assume is in the directionf

$$
T T \propto\left(\begin{array}{cc}
X^{3 / 4} \epsilon & 0 \\
0 & 0
\end{array}\right), \quad \epsilon=\left(\begin{array}{cc}
0 & 1 \\
-1 & 0
\end{array}\right),
$$

where we use the basis

$$
T=\left(\begin{array}{c}
T^{11} \\
T^{12} \\
\vdots \\
T^{42}
\end{array}\right)
$$

The field $X$ parameterizes the flat direction. A VEV for $X$ breaks the conformal symmetry, so $X$ is the Nambu-Goldstone mode for spontaneous breaking of scale symmetry. We have defined $X$ so that it has dimensions of mass.

The first threshold in this theory is given by the $\operatorname{VEV}\langle X\rangle$, where the conformal symmetry is spontaneously broken. NDA tells us that the physical threshold is at a scale $\sim(\rho\langle\tilde{T}\rangle)^{4 / 3}$, and that the canonically normalized modulus field is $X \sim \rho^{1 / 3} \tilde{T}^{4 / 3}$. The effective lagrangian below the scale of conformal symmetry breaking is written in terms of the modulus $X$ and the $\mathrm{SQCD}_{3}$ fields:

$$
\begin{aligned}
\mathcal{L}_{\text {eff }}(\mu \lesssim \rho|X|) \sim \int & d^{4} \theta\left\{[1+\Delta \ln Z(\mu \sim \rho|X|)] X^{\dagger} X+Z_{P, 0}\left(P^{\dagger} P+\bar{P}^{\dagger} \bar{P}\right)\right\} \\
& +\int d^{2} \theta \frac{\lambda}{\rho^{1 / 2} M^{1 / 2}} X^{3 / 2} \bar{P} P+\text { h.c. } \\
& +S U(3) \text { gauge kinetic terms. }
\end{aligned}
$$

The superpotential in Eq. (4.3) gives rise to a mass for the $P$ fields

$$
m_{P} \sim \frac{\lambda}{\rho^{1 / 2} M^{1 / 2}}\langle X\rangle^{3 / 2} .
$$

\footnotetext{
${ }^{5}$ For more detail on the moduli space of this theory, see Ref. [9].
} 
We consider the case $m_{P}>\Lambda_{3}$, where $\Lambda_{3}$ is the scale where the $\mathrm{SQCD}_{3}$ gauge theory with 2 flavors becomes strong, and will check the self-consistency of this choice later. In this case, we can integrate out the $P$ fields perturbatively at the scale $m_{P}$, and the effective theory is

$$
\begin{aligned}
\mathcal{L}\left(\mu \lesssim m_{P}\right) \sim \int d^{4} \theta[1 & +\Delta \ln Z(\mu \sim \rho|X|) \\
& \left.+\frac{\lambda^{2}}{\rho^{3} Z_{P, 0}^{2} M}|X| \ln \left(\frac{\rho|X|}{m_{P}}\right)\right] X^{\dagger} X
\end{aligned}
$$

$+S U(3)$ gauge kinetic terms.

The $\ln |X|$ term gives the leading effect of $P$ loops between the scales $\rho\langle X\rangle$ and $m_{P} .0$

The pure $S U(3)$ gauge theory becomes strong at a scale

$$
\Lambda_{3, \mathrm{eff}} \sim m_{P}^{2 / 9} \Lambda_{3}^{7 / 9}
$$

Gaugino condensation gives rise to an effective superpotential

$$
W_{\mathrm{dyn}} \sim \frac{\Lambda_{3, \mathrm{eff}}^{3}}{\rho^{2}} \sim \Lambda_{\mathrm{int}}^{2} X
$$

where

$$
\Lambda_{\text {int }}^{2} \sim \frac{\lambda^{2 / 3} \Lambda_{3}^{7 / 3}}{\rho^{7 / 3} M^{1 / 3}} .
$$

Eq. (4.7) is the superpotential of a Polonyi model, which breaks SUSY provided that the Kähler terms stabilize the field $X$. The vacuum energy is then of order $\Lambda_{\text {int }}^{4}$, and therefore $m_{3 / 2} \sim \Lambda_{\text {int }}^{2} / M$.

The effective potential for $X$ including the Kähler terms of Eq. (4.5) is

$$
\begin{aligned}
V_{\mathrm{eff}}=\Lambda_{\mathrm{int}}^{4} & / \frac{\partial^{2} K_{\mathrm{eff}}}{\partial X \partial X^{\dagger}}+\Delta V_{\mathrm{SUGRA}} \\
\sim \Lambda_{\mathrm{int}}^{4} & {\left[1+\left(\ln z_{0}\right)\left(\frac{\rho|X|}{M}\right)^{\beta_{*}^{\prime}}-\frac{\lambda^{2}}{\rho^{3}} \frac{|X|}{M} \ln \left(\frac{\rho|X|}{m_{P}}\right)\right] } \\
& -\Lambda_{\text {int }}^{4}(1+\operatorname{Re}(X) / M) .
\end{aligned}
$$

6 The precise coefficient of this $\log$ term is calculable but is unimportant because of the order 1 uncertainties in the other coefficients such as $m_{P}$. 
Here we have written out the leading terms in the solution for $\Delta \ln Z$ (see Eq. (3.19)) and used $(\Delta \ln Z)_{0}=\ln z_{0}$ (see Eq. (3.2)). We have dropped terms comparable to the $\ln |X|$ term that are not log-enhanced. The coefficient of the $\ln z_{0}$ term depends on strong interactions, but we can choose the sign of $\ln z_{0}$ so that the sign of this term is positive. The last line contains the leading SUGRA corrections once we add a constant superpotential so as to cancel the $\Lambda_{\text {int }}^{4}$ contribution to the cosmological constant. We will demand that the supergravity corrections to the potential dominate over the $|X| \ln |X|$ term. This gives the restriction

$$
\frac{\lambda^{2}}{\rho^{3}} \ln \left(\frac{\rho\langle X\rangle}{m_{P}}\right) \lesssim 1
$$

We then find a stable minimum at

$$
\text { sequestering } \equiv\left(\frac{\rho\langle X\rangle}{M}\right)^{\beta_{*}^{\prime}} \sim\left[\frac{1}{\rho \ln z_{0}}\right]^{\beta_{*}^{\prime} /\left(\beta_{*}^{\prime}-1\right)},
$$

where we have solved for the sequestering factor for the mixed terms in $Z_{0}$. (By Eq. (4.10), the dangerous mixed terms arising from $Z_{P, 0}$ are even more suppressed.) The term $\ln z_{0}$ parametrizes the deviation of $\mathrm{SQCD}_{2}$ from the fixed point at the Planck scale, and must be small enough that we can trust the fixed-point expansions, Eq. (3.7). NDA yields tells us that this requires $\ln z_{0} \lesssim 1$. The anomalous dimension $\beta_{*}^{\prime}$ is order 1 (and positive), and therefore the sequestering factor is an order-1 power of a loop suppression factor (up to a logarithmic correction).

In fact, there is an adjustable parameter that controls the amount of sequestering in our model. It is completely natural for the $\mathrm{SQCD}_{2}$ sector to enter the strongcoupling conformal regime at a sub-Planckian scale, $\tilde{M}<M$, although we have taken the two scales to be equal. In this more general case, we must substitute $1 / \rho \rightarrow \tilde{M} /(\rho M)$ on the right-hand side of Eq. (4.11). We can therefore obtain any desired amount of sequestering by taking $\tilde{M} \ll M$. Our analysis assumed that $\Lambda_{3}<m_{P}$, so that the $P$ 's were integrated out of the theory before the $\mathrm{SQCD}_{3}$ subsector became strongly coupled. This naturally occurs for sufficiently small $\Lambda_{3}$, which also sets the SUSY breaking scale according to Eq. (4.8). At the qualitative level, these observations show that the model naturally breaks SUSY far below the Planck scale and generates a large amount of sequestering. In the next section we will see that quantitatively, we must saturate the inequalities Eq. (4.10), $\Lambda_{3}<m_{P}$, and $\ln z_{0} \lesssim 1$ in order to get maximal sequestering for the real world. It is also optimal to take $\tilde{M} \sim M$ as we have throughout the paper. 


\section{Numerical Estimates}

We now turn to the numerical estimates in this model. Using Eq. (4.8) and Eq. (4.11) (with $\ln z_{0} \sim 1$ ) to eliminate the dependence on $\Lambda_{3}$ and $\langle X\rangle$, the constraint $\Lambda_{3}<m_{P}$ can be written

$$
\text { (sequestering })^{7 / 2} \gtrsim \frac{\rho^{7 / 2}}{\lambda^{3}}\left(\frac{\Lambda_{\text {int }}}{M}\right)^{2} .
$$

We see that we obtain maximal sequestering by saturating the bound Eq. (4.10). We will approximate the logarithm in Eq. (4.10) as order one. Note that the resulting $\lambda \sim \rho^{3 / 2}$ is smaller than the strong-coupling value, $\lambda_{\text {strong }} \sim \rho^{2}$. Substituting into Eq. (5.1) then gives a bound on the sequestering factor:

$$
\text { sequestering } \gtrsim \frac{1}{\rho^{2 / 7}}\left(\frac{\Lambda_{\text {int }}}{M}\right)^{4 / 7} \sim 6 \times 10^{-5} .
$$

We have taken $M=2.4 \times 10^{18} \mathrm{GeV}$ and $\Lambda_{\text {int }} \simeq 3 \times 10^{11} \mathrm{GeV}$. By Eq. (4.11), this maximal level of sequestering is obtained for $\beta_{*}^{\prime} \simeq 1.2$. The minimum is at $\langle X\rangle \sim 10^{14} \mathrm{GeV}$, and the mass of $X$ is of order $5 \times 10^{6} \mathrm{GeV}$.

The amount of sequestering is sufficient for AMSB to dominate in the visible sector, and is within an order of magnitude of the sequestering factor $3 \times 10^{-6}$ [9] [16] required to adequately suppress $\mathrm{CP}$-conserving flavor violation in anomaly-mediated SUSY breaking if the coefficients $c$ of Eq. (2.2) are of order 1. Given the considerable uncertainties in the strong-interaction coefficients, our maximal sequestering could easily be at or below this flavor-violation bound. Of course it is also possible that the c's of Eq. (2.2) are of order 1/10.

We now consider briefly the cosmology of this model. In general, models of the hidden sector suffer from the Polonyi problem [17]. Briefly stated, the problem is that models with moduli generally have a cosmological epoch where coherent oscillations of the moduli dominate the energy density of the universe, and the interactions of the moduli with the visible sector are too weak to reheat the universe to a sufficiently high temperature to allow nucleosynthesis. In the present model, this problem is less severe than in standard hidden sector models because the mass of the modulus is large compared to the weak scale and the self-interactions of the moduli are much stronger than gravitational strength. We will leave a full analysis of this issue for future work.

Another cosmological issue is the fact that the minimum we have found is a false vacuum. There is a supersymmetric vacuum at the origin $T=0$, but because $\langle X\rangle \gg \Lambda_{\text {int }}$, the tunnelling rate is suppressed by a large exponent and is cosmologically safe [18]. 


\section{Discussion and Conclusions}

It is remarkable that the simple four-dimensional model of the hidden sector presented here dynamically breaks supersymmetry and sequesters itself from the visible sector, naturally allowing anomaly mediation to dominate visible sector supersymmetry breaking. We believe that similar mechanisms of sequestering and dynamical supersymmetry breaking can occur in a large class of models, although it is difficult to check this outside of supersymmetric QCD because of the limited number of superconformal theories that are known explicitly.

According to our estimates, CP conserving flavor-changing neutral current processes are near their experimental limits. Given the large uncertainties, it is possible that there is more sequestering than given in our estimates, so that CP-violating flavor violation is also sufficiently suppressed. Alternatively, suppressing CP violating flavor violation may require additional structure. In any case, we expect some flavor-changing neutral current processes to be close to their experimental limits.

We hope that this work will help open new directions for constructing complete, compelling, and realistic hidden sector models of supersymmetry breaking.

\section{Acknowledgements}

We thank the Fermilab theory group for hospitality during the initial stage of this work. M.A.L. was supported by NSF grant PHY-98-02551. R.S. was supported in part by a DOE Outstanding Junior Investigator award DEFG0201ER41198 and in part by NSF Grant PHY-0099468. 


\section{References}

[1] L. Randall, R. Sundrum, Nucl. Phys. B557 79 (1999), hep-th/9810155.

[2] G.F. Giudice, M.A. Luty, H. Murayama, R. Rattazzi, JHEP 9812027 (1998), hep-ph/9810442.

[3] A. Pomarol and R. Rattazzi, JHEP 9905013 (1999), hep-ph/9903448.

[4] Z. Chacko, M.A. Luty, I. Maksymyk, E. Pontòn, JHEP 0004001 (2000), hep-ph/9905390; E. Katz, Y. Shadmi, Y. Shirman JHEP 9908015 (1999), hep-ph/9906296; K. I. Izawa, Y. Nomura, T. Yanagida, Prog. Theor. Phys. 1021181 (1999), hep-ph/9908240; M. Carena, K. Huitu, T. Kobayashi, Nucl. Phys. B592 164 (2000), hep-ph/0003187; B.C. Allanach, A. Dedes, JHEP 0006017 (2000), hep-ph/0003222; I. Jack, D.R.T. Jones, Phys. Lett. B491 151 (2000), hep-ph/0006116; D.E. Kaplan, G.D. Kribs, JHEP 0009048 (2000), hep-ph/0009195. N. Arkani-Hamed, D. E. Kaplan, H. Murayama, Y. Nomura, JHEP 0102041 (2001), hep-ph/0012103; T. Gherghetta, A. Riotto, hep-th/0110022.

[5] M.A. Luty, R. Sundrum, Phys. Rev. D62 035008 (2000), hep-th/9910202.

[6] M.A. Luty, R. Sundrum, Phys. Rev. D64 065012 (2001) hep-th/0012158.

[7] J. Maldacena, Adv. Theor. Math. Phys. 2231 (1998), hep-th/9711200; S.S. Gubser, I.R. Klebanov, A.M. Polyakov, Phys. Lett. B428 105 (1998), hep-th/9802109; E. Witten, Adv. Theor. Math. Phys. 2 253 (1998), hep-th/9802150.

[8] H. Verlinde, Nucl. Phys. B580 264 (2000), hep-th/9906182; J. Maldacena, unpublished remarks; E. Witten, ITP Santa Barbara conference 'New Dimensions in Field Theory and String Theory,', http://www.itp.ucsb.edu/online/susy c99/discussion; S. S. Gubser, Phys. Rev. D63 084017 (2001), hep-th/9912001; E. Verlinde, H. Verlinde, JHEP 0005034 (2000), hep-th/9912018; N. ArkaniHamed, M. Porrati, L. Randall, JHEP 0108017 (2001), hep-th/0012148; R. Rattazzi, A. Zaffaroni, JHEP 0104 (2001) 021, hep-th/0012248; M. PerezVictoria, JHEP 0105064 (2001) hep-th/0105048.

[9] M.A. Luty, R. Sundrum, hep-th/0105137, to be published in Phys. Rev. D.

[10] A.E. Nelson, M.J. Strassler, hep-ph/0104051; JHEP 0009030 (2000), hep-ph/0006251. 
[11] N. Seiberg, Nucl. Phys. B435 129 (1995), hep-th/9411149.

[12] V.A. Novikov, M.A. Shifman, A.I. Vainshtein, V.I. Zakharov, Nucl. Phys. B229 381 (1983); M.A. Shifman, A.I. Vainshtein, Nucl. Phys. B277 456 (1986); Nucl. Phys. B359 571 (1991); N. Arkani-Hamed, H. Murayama, JHEP 0006030 (2000), hep-th/9707133.

[13] N. Arkani-Hamed, G. Giudice, M.A. Luty, R. Rattazzi, Phys. Rev. D58 115005 (1998), hep-ph/9803290.

[14] A. Manohar, H. Georgi, Nucl. Phys. B234 189 (1984); H. Georgi, L. Randall, Nucl. Phys. B276 241 (1986).

[15] M.A. Luty, Phys. Rev. D57, 1531 (1998), hep-ph/9706235; A.G. Cohen, D.B. Kaplan, A.E. Nelson, Phys. Lett. 412B, 301 (1997), Phys. Rev. D59, 035005 (1999), hep-ph/9706275; L. Randall, R. Rattazzi, E. Shuryak, Phys. Rev. D59, 035005 (1999), hep-ph/9803258.

[16] F. Gabbiani, E. Gabrielli, A. Masiero, L. Silvestrini, Nucl. Phys. B477 321 (1996), hep-ph/9604387.

[17] J. Polonyi, Budapest preprint KFKI-93 (1977); G.D. Coughlan, R. Holman, P. Ramond, and G.G. Ross, Phys. Lett. B140 44 (1984); B. de Carlos, J.A. Casas, F. Quevedo, and E. Roulet, Phys. Lett. B318 447 (1993); T. Banks, D. B. Kaplan, and A. Nelson, Phys. Rev. D49 779 (1994).

[18] K.-Y. Lee, E.J. Weinberg, Nucl. Phys. B267, 181 (1986); S. Dimopoulos, G. Dvali, R. Rattazzi, G.F. Giudice, Nucl. Phys. B510 12 (1998), hep-ph/9705307. 